\title{
Emerging Therapeutic Options in Acute Lymphoblastic Leukemia
}

\author{
Presented by Patrick A. Brown, MD
}

\begin{abstract}
Immunotherapies have dramatically increased response rates in the relapsed/refractory setting of acute lymphoblastic leukemia. These emerging therapeutic options include blinatumomab, a bispecific T-cell engager construct; inotuzumab, an antibody-drug conjugate; and CAR T cells. Despite significantly improved rates of response, however, CAR T-cell therapy is the only approach associated with durable survival in a significant proportion of patients. Immunotherapies come with characteristic toxicity profiles. Inotuzumab is associated with hepatotoxicity, and blinatumomab and CAR T cells are associated with both cytokine release syndrome and neurotoxicity. Furthermore, immunotherapy is not always successful. Several mechanisms of failure exist, including failure to manufacture the CAR product, failure to engraft or lack of persistence of CAR T cells, endogenous T cell or CAR T-cell exhaustion, and antigen escape.
\end{abstract}

Acute lymphoblastic leukemia (ALL) is the most common cancer in children, but occurs across the age spectrum. Prognosis varies greatly by age, with cure rates ranging from $90 \%$ in children to only $40 \%$ in older adults. For patients who develop relapsed or refractory disease, various salvage therapies are available, but immunotherapies have produced a paradigm shift in response rates. At the NCCN 2020 Virtual Congress: Hematologic Malignancies, Patrick A. Brown, MD, Professor of Oncology, The Sidney Kimmel Comprehensive Cancer Center at Johns Hopkins University, discussed emerging therapeutic options in relapsed/refractory B-cell ALL, including blinatumomab, inotuzumab, and chimeric antigen receptor (CAR) $\mathrm{T}$ cells.

\section{Diagnosis and Treatment of ALL}

As Dr. Brown reported, the standard treatment of ALL is risk-stratified using a combination of clinical, molecular, and early response features. Initial treatment typically lasts several months and includes remission induction chemotherapy, central nervous system prophylaxis and treatment, and consolidation chemotherapy (Figure 1). Then, depending on risk factors, therapy continues with either maintenance chemotherapy for another 1 to 2 years or allogeneic stem cell transplant in first remission. Because ALL can occur with tyrosine kinase mutations such as BCR-ABL1, treatment may also include a tyrosine kinase inhibitor.

\section{Immunotherapy for ALL}

As Dr. Brown explained, most ALL cases (up to 95\%) will express CD19 and CD22 antigens. CD20 is often expressed in adult ALL, but less frequently in pediatric ALL. These antigens (CD19, CD20, and CD22) make good targets for ALL therapy, said Dr. Brown, because they are not expressed on bone marrow stem cells or on other tissues in the body, but are specific to B cells. "Even though we eliminate normal $\mathrm{B}$ cells in the process of targeting these antigens, the complete loss of normal B cells is something that patients can tolerate, at least for a time, through the replacement of immunoglobulins," he explained.

Inotuzumab is a CD22-directed antibody-drug conjugate linked to calicheamicin. It is administered as a 1-hour intravenous infusion weekly. Blinatumomab is a bispecific $\mathrm{T}$-cell engager construct that targets and binds to CD19 on the surface of tumor cells and CD3 on the surface of normal $\mathrm{T}$ cells. It allows recognition of tumor-associated surface antigens and acts independently of specificity of T-cell receptor. However, it relies on functional endogenous cytotoxic T-cell response, which is different from inotuzumab, which acts through a $\mathrm{T}$ cell-independent mechanism, said Dr. Brown. Blinatumomab is administered as a 28-day continuous intravenous infusion, with bag changes occurring every 4 to 7 days.

Finally, CAR T cells are at the intersection of 3 innovative technologies:

- Cellular therapy, in using the patient's own cells as therapy;

- Gene therapy, in inserting genes into a patient's cells, thereby causing these cells to produce a new therapeutic protein; and 



Figure 1. Diagnosis and treatment of acute lymphoblastic leukemia.

Abbreviations: alloSCT, allogeneic stem cell transplant; FISH, fluorescence in situ hybridization; MRD, minimal residual disease; TKI, tyrosine kinase inhibitor

- Immunotherapy, in harnessing the patient's own immune system to treat disease.

As Dr. Brown explained, delivering CAR T cells is an extremely complicated, multidisciplinary process that involves multiple stakeholders at an institution.

\section{Clinical Responses to Immunotherapy}

To date, immunotherapies have had their biggest impact in the relapsed/refractory setting, where they have produced a paradigm shift in response rates (Figure 2). ${ }^{1-4}$ Blinatumomab is associated with a $44 \%$ complete remission rate and a $33 \%$ minimal residual disease (MRD)-negative complete remission rate. ${ }^{1}$ This depth of remission was not seen with prior salvage therapies. Inotuzumab is associated with an even greater induction of complete remission and MRD-negative response, with up to $63 \%$ MRD-negative rates. ${ }^{2}$ CAR $\mathrm{T}$ cells have the highest response rates, with complete remission and MRD-negative complete remission between $80 \%$ and $90 \% .^{3}$

In the pivotal trial that led to FDA approval of blinatumomab in adults, patients were randomized to blinatumomab versus investigators' choice chemotherapy. ${ }^{4}$ Although survival was improved initially and median duration of survival was better in the blinatumomab versus chemotherapy arms, said Dr. Brown, eventually these curves met. Blinatumomab is not associated with prolonged survival in and of itself. ${ }^{4}$
The same is true of inotuzumab, Dr. Brown said. An improvement was seen in median survival, but no statistically significant improvement was seen in overall survival. ${ }^{2}$

CAR T cells are the exception with respect to survival. There does appear to be a prolonged, durable survival advantage without subsequent therapy in approximately $40 \%$ to $50 \%$ of patients. ${ }^{3}$ Although CAR T cells can represent definitive therapy in a subset of patients, said Dr. Brown, clinicians are still struggling to predict which patients those are. However, emerging biomarkers may help with patient stratification.

Blinatumomab is unique among the immunotherapies in that it is also FDA approved for patients with MRD, in whom it has demonstrated efficacy and increased tolerability relative to chemotherapy, said Dr. Brown.

\section{Toxicities From Immunotherapy}

According to Dr. Brown, inotuzumab has a unique toxicity profile, with hepatotoxicity being the primary adverse event. Hepatotoxicity can occur either before or after hematopoietic stem cell transplant. "Because inotuzumab does not represent definitive therapy and is often a bridge to transplant, this hepatotoxicity is very significant," said Dr. Brown. He noted that the primarily toxicity is hepatic sinusoidal obstruction syndrome. "This can be a major limitation of this product."

Blinatumomab and tisagenlecleucel, the first CAR T-cell product approved by the FDA, have similar adverse 


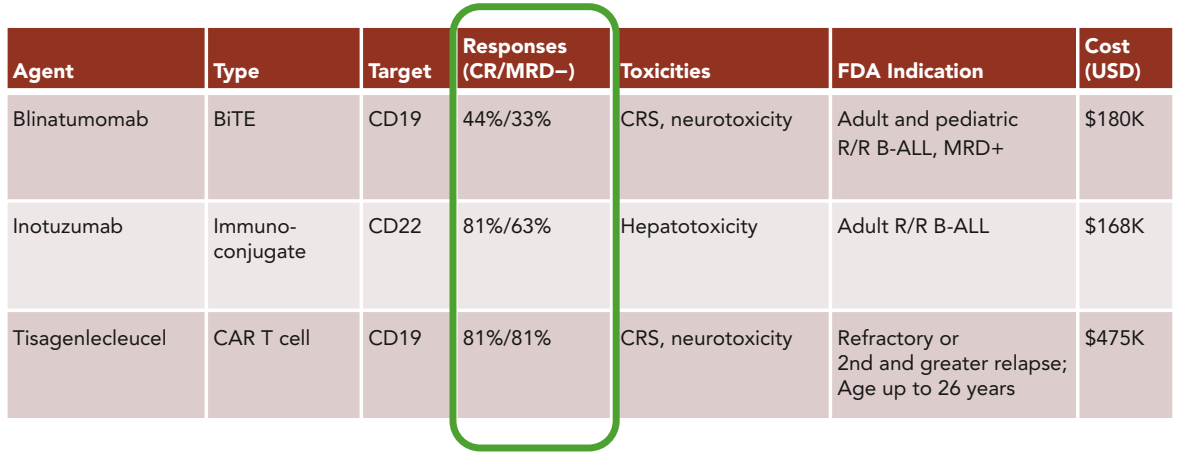

Figure 2. Response and survival rates in R/R B-ALL.

Abbreviations: B-ALL, B-cell acute lymphoblastic leukemia; BiTE, bispecific T-cell engager; CR, complete response; CRS, cytokine release syndrome; MRD, minimal residual disease; $R / R$, relapsed/refractory.

Data from references $1-4$.

event profiles, with cytokine release syndrome (CRS) and cytokine-mediated neurotoxicity being the primary toxicities. According to Dr. Brown, the timing of these 2 toxicities is dictated and predicted by the timing of CAR $\mathrm{T}$-cell expansion, or in the case of blinatumomab, endogenous T-cell expansion.

"That expansion typically occurs most rapidly within the first week of therapy, which is the high-risk period for CRS," said Dr. Brown, who noted that CRS is characterized by fever, hypotension, respiratory compromise, and potentially coagulopathy.

Neurologic toxicities are the second major adverse event, and these tend to occur later in the course of treatment, after CRS. They can occur as early as 1 week or as late as several weeks after either the beginning of blinatumomab therapy or the infusion of CAR T cells. According to Dr. Brown, neurologic toxicities take 2 major forms: encephalopathic changes, such as somnolence, confusion, and delirium; or seizures, which tend to be relatively easy to treat and reversible and can often be prevented with seizure prophylaxis. The incidence of CRS falls between $40 \%$ and $80 \%$. Blinatumomab is associated with the lower range, whereas CAR $\mathrm{T}$ cells tend to have higher rates of CRS. According to Dr. Brown, half of these cases are grade $\geq 3$ severity.

Neurologic toxicities are less frequent, occurring at a rate of approximately $10 \%$ to $30 \%$, and rates of grade $\geq 3$ severity are only $5 \%$ to $10 \%$. Interestingly, said Dr. Brown, even though CRS and neurotoxicity are both mediated by cytokines, they may not correlate with each other. Patients may have very significant CRS but no neurotoxicity, and vice versa.

"It's important to understand that the chances of developing serious CRS correlates with disease burden at the time of treatment," said Dr. Brown. "Patients with bulky disease treated in the relapsed/refractory setting have a high incidence of CRS and a high incidence of serious CRS, whereas patients with low disease burden treated in an MRD setting have significantly lower rates and severity of CRS." Conversely, the rates and severity of neurotoxicity do not appear to correlate with the disease burden at the time of treatment.

\section{Mechanisms of Failure of Immunotherapy}

Unfortunately, said Dr. Brown, immunotherapy does not always work; there are a number of mechanisms by which they may fail patients. With CAR $\mathrm{T}$ cells specifically, there can be a failure to manufacture the CAR product. For example, physicians may have difficulty in getting enough functional $\mathrm{T}$ cells in younger patients to create the product. In addition, in patients who have been heavily pretreated, said Dr. Brown, endogenous $\mathrm{T}$ cells may not be sufficient in number or function to create the CAR T-cell product.

The second potential failure of immune therapy is failure of the CAR T cells to engraft or failure to persist in patients if they do engraft. A number of optimizations are being studied to overcome this limitation, such as testing different costimulatory domains that may be more effective in allowing the CAR T cell to expand or persist. Coinfusion of antigen-presenting cells has also been studied, with some evidence of efficacy. Finally, there are CAR-T cell products that use fully humanized gene sequences to avoid the production of antimurine antibodies.

Another problem with both blinatumomab and CAR T cells is CAR T-cell "exhaustion," which is failure of $\mathrm{T}$ cells to respond appropriately to presenting antigens. According to Dr. Brown, this mechanism of resistance can potentially be overcome therapeutically with the use of checkpoint inhibitors, and multiple clinical trials are seeking to combine checkpoint inhibitors with either blinatumomab or CAR T cells. 
Finally, the last mechanism of resistance that can occur is antigen escape, which has been demonstrated with CD19-targeted therapies. According to Dr. Brown, the incidence of antigen escape is lower with blinatumomab than with CAR T cells, but that simply reflects the lower potency of blinatumomab in targeting CD19. Researchers are developing multi-antigen-targeted approaches to simultaneously target several of the antigens on the surface of the leukemic cells.
"These approaches are in clinical trials and hopefully will make an impact down the road," Dr. Brown concluded.

Disclosures: Dr. Brown has disclosed that he has received honoraria from Aptitude Health and Novartis Pharmaceuticals Corporation, and consulting fees from Jazz Pharmaceuticals Inc., Novartis Pharmaceuticals Corporation, and Servier.

Correspondence: Patrick A. Brown, MD, Johns Hopkins Kimmel Cancer Center, 1650 Orleans Street, CRB1 Room 2M51, Baltimore, MD 21231. Email: pbrown2@jhmi.edu

\section{References}

1. Topp MS, Gökbuget N, Stein AS, et al. Safety and activity of blinatumomab for adult patients with relapsed or refractory B-precursor acute lymphoblastic leukaemia: a multicentre, single-arm, phase 2 study. Lancet Oncol 2015;16:57-66

2. Kantarjian HM, DeAngelo DJ, Stelljes M, et al. Inotuzumab ozogamicin versus standard therapy for acute lymphoblastic leukemia. N Engl J Med 2016:375:740-753

3. Maude SL, Laetsch TW, Buechner J, et al. Tisagenlecleucel in children and young adults with B-cell lymphoblastic leukemia. N Engl J Med 2018;378: 439-448.

4. Kantarjian H, Stein A, Gökbuget N, et al. Blinatumomab versus chemotherapy for advanced acute lymphoblastic leukemia. N Engl J Med 2017 376:836-847. 